\title{
Impact of F1Fo-ATP-synthase dimer assembly factors on mitochondrial function and organismic aging
}

\author{
Nadia G Rampello', Maria Stenger ${ }^{2}$, Benedikt Westermann ${ }^{2}$, Heinz D Osiewacz ${ }^{1, *}$ \\ ${ }^{1}$ Department of Biosciences, Molecular Developmental Biology, Institute of Molecular Biosciences and Cluster of Excellence \\ Frankfurt Macromolecular Complexes, J. W. Goethe University, 60438 Frankfurt, Germany. \\ ${ }^{2}$ Cell Biology and Electron Microscopy, University of Bayreuth, 95440 Bayreuth, Germany. \\ * Corresponding Author: \\ Heinz D. Osiewacz, Tel: +496979829264; E-mail: Osiewacz@bio.uni-frankfurt.de
}

\begin{abstract}
In aerobic organisms, mitochondrial $\mathrm{F}_{1} \mathrm{~F}_{\mathrm{o}}$-ATP-synthase is the major site of ATP production. Beside this fundamental role, the protein complex is involved in shaping and maintenance of cristae. Previous electron microscopic studies identified the dissociation of $F_{1} F_{0}$-ATP-synthase dimers and oligomers during organismic aging correlating with a massive remodeling of the mitochondrial inner membrane. Here we report results aimed to experimentally proof this impact and to obtain further insights into the control of these processes. We focused on the role of the two dimer assembly factors PaATPE and PaATPG of the aging model Podospora anserina. Ablation of either protein strongly affects mitochondrial function and leads to an accumulation of senescence markers demonstrating that the inhibition of dimer formation negatively influences vital functions and accelerates organismic aging. Our data validate a model that links mitochondrial membrane remodeling to aging and identify specific molecular components triggering this process.
\end{abstract}

doi: $10.15698 /$ mic2018.04.625 Received originally: 28.11.2017; In revised form: 23.01.2018, Accepted 25.01.2018, Published 30.01.2018.

Keywords: aging, F1Fo-ATPsynthase, membranes, mitochondria, remodeling.
Abbreviations:
$\mathrm{CJ}$-cristae junction,
IM-inner membrane,
MICOS - mitochondrial contact site
and cristae organizing system,
MPTP - mitochondrial permeability
transition pore,
mtDNA - mitochondrial DNA,
OM-outer membrane,
OXPHOS-oxidative phosphorylation,
$P C D$ - programmed cell death.

\section{INTRODUCTION}

Mitochondria are eukaryotic organelles involved in a number of essential processes including the synthesis of iron/sulfur clusters, biosynthesis of lipids and amino acids, energy transduction and generation of ATP. In aerobic organisms, most of this universal cellular energy carrier is generated by oxidative phosphorylation (OXPHOS) at the respiratory chain. The protein complexes and supercomplexes of the respiratory chain are located in invaginations of the inner membrane (IM), the so-called cristae, which occur in different shapes (e.g. tubular, lamellar) [1-4]. At their basis, the cristae junctions (CJ), cristae are connected to the outer membrane (OM), which surrounds the organelle.

$\mathrm{F}_{1} \mathrm{~F}_{\mathrm{o}}$-ATP-synthase is an IM-bound protein complex that is essential for the electrochemical-driven generation of ATP. The individual proteins of the complex are encoded by the nuclear and by mitochondrial DNA (mtDNA). Mutations in the corresponding genes lead to $F_{1} F_{0}$-ATP-synthase deficiency, mitochondrial dysfunction [5] and severe neurodegenerative pathologies like Alzheimer's and Parkinson's disease [6-8] and have a strong impact on biological aging [9-11]. Beside its enzymatic role, the $F_{1} F_{0}-A T P-s y n t h a s e$ complex plays a key role in shaping cristae structure [1214]. In particular, rows of $F_{1} F_{0}$-ATP-synthase dimers at the tips of the cristae appear to be critical for maintaining their typical convex curvature $[15,16]$. Recent studies identified the impact of another protein complex, the mitochondrial contact site and cristae organizing system (MICOS) [17], in controlling IM ultrastructure $[3,18-20]$. This complex is localized at the CJ. Despite the distant localization of the two complexes, interactions between them, or at least of components of them, were demonstrated. It was found that Mic10, a core component of MICOS, interacts with the dimeric $\mathrm{F}_{1} \mathrm{~F}_{\mathrm{o}}$-ATP-synthase and stabilizes the complex [21, 22]. The mechanisms regulating these interactions are 
largely unknown but are of key relevance for the remodeling of mitochondrial ultrastructure that is observed as adaptive response to physiological changes [23, 24].

Pronounced changes of the mitochondrial ultrastructure were previously demonstrated to occur during aging of the aging model organism Podospora anserina. Mitochondria from juvenile cultures predominantly contain well-structured lamellar cristae with rows of $F_{1} F_{0}$-ATPsynthase dimers at their tips. During aging, the invaginations of the IM recede and the membrane curvature becomes concave forming a system of vesicles in the matrix space. This process of membrane reorganization goes along with a dissociation of $F_{1} F_{0}$-ATP-synthase dimers. Occasionally, vesicles in the matrix of old mitochondria are found in close contact with the OM at contact sites. It was suggested that at late age the OM bursts at these sites and releases mitochondrial content to the cytosol inducing programmed cell death (PCD) [25]. The relevance of this series of cellular events observed during $P$. anserina aging is supported by genetic manipulations of the molecular machinery involved in the control of PCD, the final phase in the life cycle of $P$. anserina $[26,27]$. Strikingly, overexpression of a gene coding for the mitochondrial peptidyl prolylcis, trans-isomerase, cyclophilin D (CYPD), which is involved in the control of the mitochondrial permeability transition pore (mPTP) opening [28], leads to accelerated aging. In this overexpression strain mitochondria of young chronological age (6-days) display a vesicular ultrastructure that is characteristic for old wild-type mitochondria of 18-days of age [29]. The molecular components involved in this process and its regulation were unknown.
In the yeast Saccharomyces cerevisiae, it was shown that dimer and oligomer formation is mediated by the interaction of two dimer assembly factors, subunit e (Atp21, Su e) and subunit $g$ (Atp20, Su g) of $F_{1} F_{0}$-ATP-synthase [30, 31]. Deletion of a gene coding for either one of these proteins leads to a loss of dimers and oligomers without influencing the catalytic ATP-synthase activity of the enzyme but inducing a strong alteration of mitochondrial ultrastructure $[30,32]$. Surprisingly, the downregulation of the gene coding for subunit $e$ or $g$ in HeLa cells affects not only the stability of dimers and oligomers but also the catalytic activity of the enzyme and mitochondrial morphology [33].

Here we report novel data from a study aimed to generate mechanistic insights into the impact of mitochondrial ultrastructure on biological aging. We focused on PaAtpe and PaAtpg encoding the two putative $\mathrm{F}_{1} \mathrm{~F}_{\mathrm{o}}$-ATP-synthase dimer assembly factors of $P$. anserina and investigated their age-related expression. Moreover, we analyzed the impact of the ablation of the two dimer assembly factors on biological aging in detail. Our data extend a model describing the age-related mitochondrial membrane remodeling including the role of $F_{1} F_{0}$-ATP-synthase dimers and dimer assembly factors PaATPE and PaATPG.

\section{RESULTS AND DISCUSSION}

The abundance of $F_{1} F_{0}$-ATP-synthase dimers decreases during aging

Previous work revealed an essential role of $F_{1} F_{0}$-ATPsynthase dimers in the formation of mitochondrial cristae $[12-14,16]$ and a decrease of mitochondria with lamellar cristae accompanied by an increase of those with a vesicu-
A

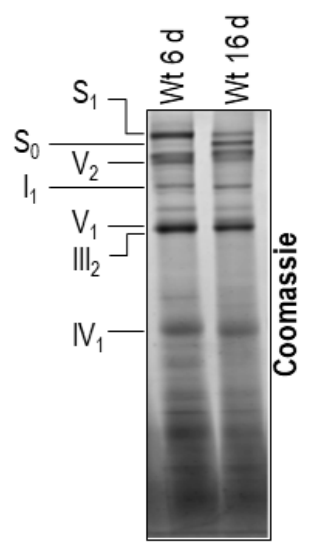

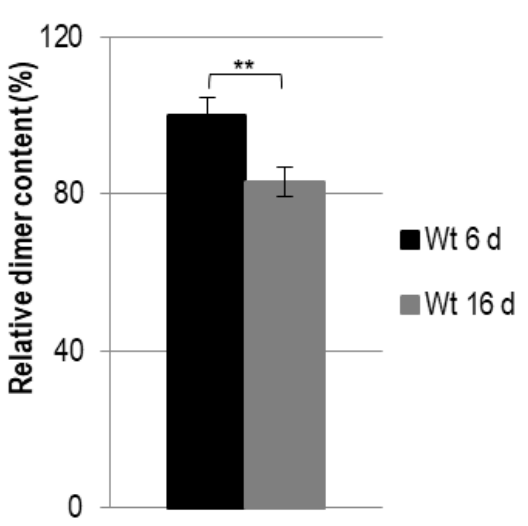

B

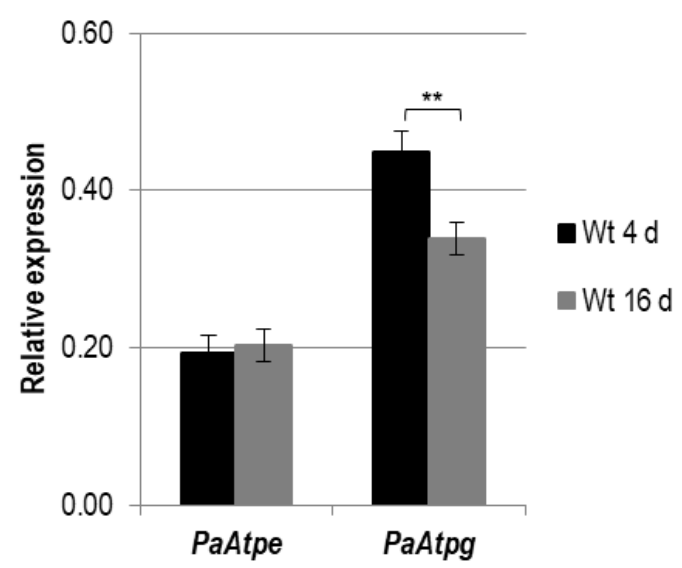

FIGURE 1: Age-dependent dimer decrease and analysis of expression of PaAtpe and PaAtpg in P. anserina. (A) Representative BN-PAGE analysis and quantification of mitochondrial protein extracts (V2, $F_{1} F_{0}$-ATP-synthase dimers) from three independent 6-days and 16-days old wild-type strains. $F_{1} F_{0}$-ATP-synthase dimers were normalized to Coomassie stained gel. Relative dimer content of 6-days old wild-type strains was set to $100 \%$. Mitochondrial protein complexes were stained with Coomassie Blue and complexes and supercomplexes $\mathrm{S}_{1}$, $\mathrm{S}_{0}$, $\mathrm{V}_{2}$ $\mathrm{I}_{1}, \mathrm{~V}_{1}, \mathrm{III}_{1}$ and $\mathrm{IV}_{1}$ are indicated. (B) Transcript analysis of PaAtpe and PaAtpg in three young (4 d) and old (16 d) wild type cultures, respectively. RNA was isolated and gene expression was determined by qRT-PCR analyses. The relative expression was normalized to the expression of PaPorin coding for a mitochondrial outer membrane protein. Error bars represent the standard deviation and the P-values were determined by Student's t test. 
lar ultrastructure during aging of $P$. anserina $[25,29]$. To elucidate the biochemical basis of these microscopic observations, we analyzed mitochondria from batches isolated from 6-days and 16-days old cultures of the $P$. anserina wild type that previously were used for cryo-electron microscopy [25] and subjected them to BN-PAGE analysis (Fig. 1A). We observed a $20 \%$ decrease of $F_{1} F_{0}$-ATP-synthase dimers in 16-days old cultures compared to 6-days old cultures. This effect is likely to be underestimated because of the elimination of impaired mitochondria during aging by autophagy, a quality control pathway that increases during aging $[34,35]$. Apart from a decrease in abundance of $F_{1} F_{0}-$ ATP-synthase dimers, a marked change of supercomplex amount $S_{1}\left(I_{1} I I_{2} I V_{1}\right)$ and $S_{0}\left(I_{1} I I_{2} I V_{0}\right)$ is observed during aging (Fig. 1A). In cells of 6 -days old cultures $S_{1}$ predominates while this supercomplex is decreased in 16-days cultures. In contrast, an increase of $S_{0}$ is observed during aging (Fig. 1A).

Next, we investigated the age-associated expression of PaAtpe and PaAtpg, coding for two potential factors involved in the control of $F_{1} F_{0}$-ATP-synthase dimer formation. A comparative transcript analysis of these genes of young (4-days) and senescent (16-days) $P$. anserina cultures revealed a reduction of the PaAtpg transcript level but no significant alteration of PaAtpe transcripts (Fig. 1B), suggesting that the age-related regulation or stability of the two putative dimer-specific genes differs. Interestingly, in the young wild type, transcript levels of PaAtpe are considerably lower than those of PaAtpg. These observations let us speculate about different regulatory mechanisms and probably different functions of the two dimer assembly factors. It appears that PaATPE is a limiting but central factor in dimer formation. This idea is supported by different studies with yeast demonstrating that dimer assembly factors bind to the $F_{0}$ part of ATP-synthase in a sequential manner. First Su e binds to the $F_{0}$ part followed by the association of Su $g[31,36]$. It was shown that the presence of Su e is required for stabilization of Su g whereas Su $g$ is not necessary for binding of Su e [37]. Thus, Su e appears to play a key role in ATP-synthase dimer formation while Su g acts as a protein supporting Su e in this function [30, 31].

Overall, the observed changes in $\mathrm{F}_{1} \mathrm{~F}_{\mathrm{o}}$-ATP-synthase dimer quantity and alterations of supercomplexes $S_{1}$ to $S_{0}$ indicate mitochondrial membrane reorganization processes during aging of $P$. anserina. These biochemical data are in concordance with the observations of earlier cryoelectron microscopy studies $[25,29]$.

\section{Ablation of PaATPE and PaATPG affects dimer formation and mitochondrial ultrastructure}

Next, we focused on the role of PaATPE and PaATPG in the formation of $F_{1} F_{0}-A T P$-synthase dimers. In yeast and HeLa cells the lack of one of the homologues is sufficient to inhibit $F_{1} F_{0}$-ATP-synthase dimer formation $[30,31,33]$. In both model organisms, the deletion of either gene strongly affects mitochondrial ultrastructure. In yeast, onion-like structures of the IM are found [12]. In HeLa cells, the IM forms arch-like or longitudinal cristae [33].
We generated $P$. anserina strains in which either PaAtpe ( $P$. anserina accession number: Pa_1_3740) or PaAtpg ( $P$. anserina accession number: $P a \_1 \_2480$ ) were replaced by a bifunctional selection marker that mediates phleomycin and blasticidin resistance [38]. Strains were verified by Southern blot analysis using a specific Phleo and PaAtpe or PaAtpg probe (Fig. 2A). Subsequent analysis of mitochondrial extracts from each of the two deletion strains by BN-PAGE revealed the absence of $F_{1} F_{0}$-ATPsynthase dimers (Fig. 2B) and demonstrated that the $P$. anserina proteins encoded by these two genes are indeed involved in the dimerization of $F_{1} F_{0}$-ATP-synthase monomers.

Subsequently, we analyzed whether the lack of $F_{1} F_{0}$ ATP-synthase dimers affects mitochondrial ultrastructure. Electron microscopy of mitochondria isolated from young (6-days) and old (16-days) wild-type strains revealed pronounced remodeling of the IM during aging (Fig. 2C), corresponding to what was described earlier in a cryo-electron microscopy study [29]. While well-separated lamellar cristae with a typical convex curvature at the tips could be readily found in preparations of young wild type mitochondria, mitochondria from old cultures were generally devoid of lamellar cristae and the inner membrane frequently formed septa and vesicular structures (reticulate ultrastructure). Mitochondria isolated from PaAtpe and PaAtpg deletion strains of young chronological age (i.e. 6days) strikingly resemble old wild type mitochondria (16days). Importantly, typical lamellar cristae could not be found in mutant or old wild type mitochondria (Fig. 2C). These results clearly demonstrate a direct impact of $\mathrm{F}_{1} \mathrm{~F}_{\mathrm{O}^{-}}$ ATP-synthase dimers on the formation of lamellar cristae and aging-dependent mitochondrial membrane reorganization.

Defects in $F_{1} F_{0}$-ATP-synthase dimerization affects mitochondrial function and leads to accelerated aging

In a series of subsequent experiments, we concentrated on the impact of $\mathrm{F}_{1} \mathrm{~F}_{\mathrm{o}}$-ATP-synthase dimerization on aging of $P$. anserina. We compared well-known senescent markers in the two deletion strains and the wild type of defined chronological age. Apart from the recently identified ultrastructural reorganization of the IM, changes in mitochondrial morphotype [39], reorganization of the mitochondrial DNA (mtDNA) [40-44], an increase in hydroxide peroxide $\left(\mathrm{H}_{2} \mathrm{O}_{2}\right)$ release from cultures $[39,45]$ and impairments in respiration [46] were analyzed as hallmarks of aging.

Light microscopy analysis identified an accelerated change of mitochondrial morphotypes in the two investigated deletion strains. In contrast to the wild type, in which mitochondria from 3-days old cultures are mainly of a filamentous morphotype, mitochondria of the same chronological age of the two deletion mutants display a punctate morphotype that is characteristic for mitochondria of old (i.e. 16-days old) wild-type strains (Fig. 3A).

Analysis of mtDNA revealed an accelerated amplification of a mitochondrial plasmid-like DNA (pIDNA). In 4-days old PaAtpe and PaAtpg deletion strains this senescent marker is already strongly amplified. In the wild type, 
A

B

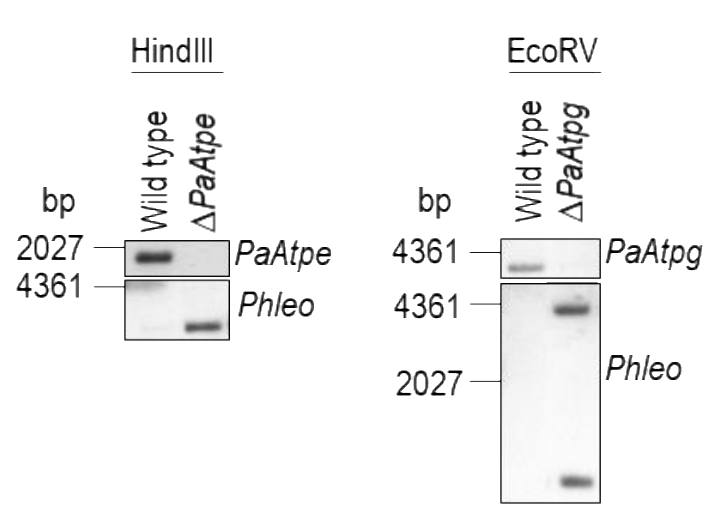

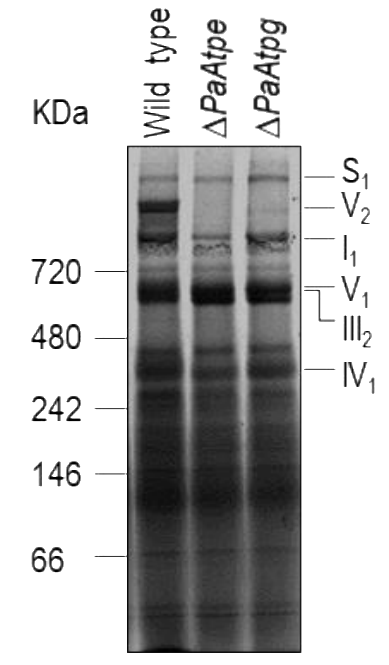

C

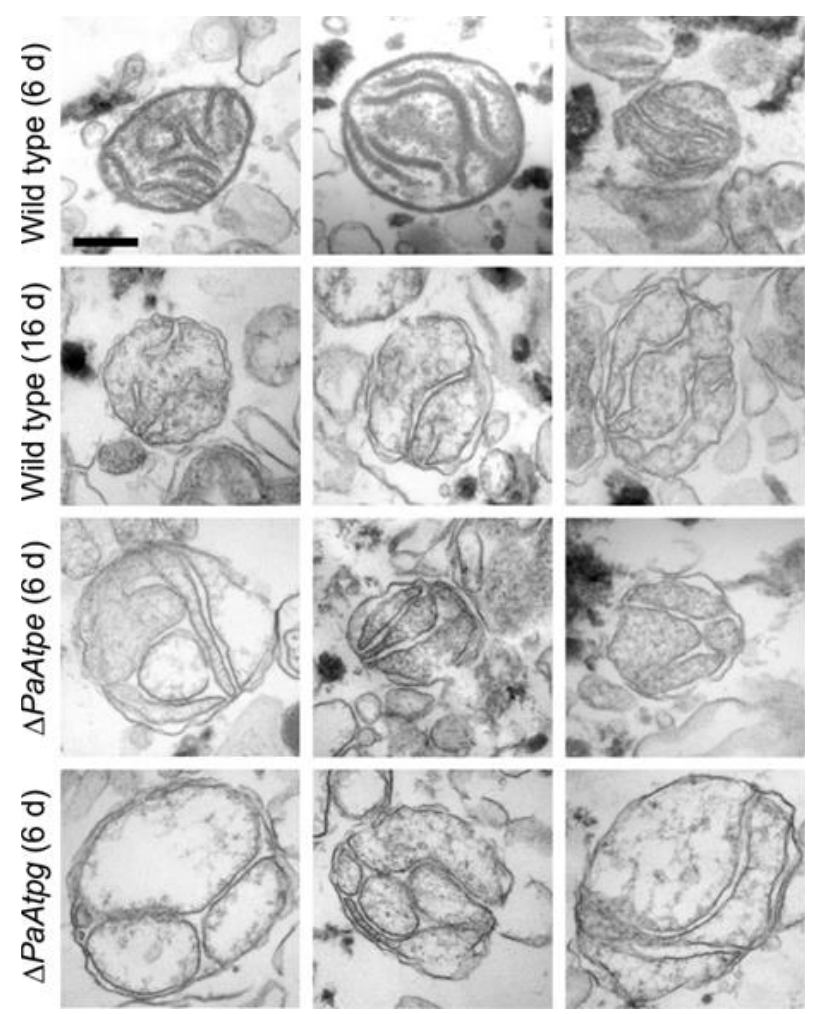

FIGURE 2: Deletion of PaAtpe or PaAtpg affects $\mathrm{F}_{1} \mathrm{~F}_{\mathrm{o}}$-ATP-synthase dimer formation and mitochondrial inner membrane ultrastructure. (A) Southern blot analysis of HindIII- and EcoRV-digested genomic DNA (gDNA) of wild-type, $\triangle$ PaAtpe and $\triangle$ PaAtpg strains. The digested gDNA was hybridized with a specific PaAtpe, PaAtpg and Phleo probe, respectively, and demonstrated a successful deletion of PaAtpe and PaAtpg. (B) Representative BN-PAGE analysis of mitochondrial protein extracts from 6-days old wild-type, $\triangle$ PaAtpe and $\triangle$ PaAtpg strains. Mitochondrial complexes including $S_{1}, V_{2}, I_{1}, V_{1}$, $\mathrm{III}_{1}$ and IV $\mathrm{V}_{1}$ were visualized by Coomassie staining. (C) EM of isolated mitochondria of wild-type and deletion strains of different age (6 days and 16 days old). The morphology of at least 50 organelles per strain and growth condition was analyzed. Representative images are shown at the same magnification. Scale bar: 0,2 $\mu \mathrm{m}$.
pIDNA is almost absent from 4-days old cultures and found in strains of much older age (e.g. 16-days) (Fig. 3B).

Next, we analyzed $\mathrm{H}_{2} \mathrm{O}_{2}$ release [39]. Incubation of wild-type and deletion strains of the same chronological age (6-days) with a diaminobenzidin (DAB) staining solution revealed an increase of a brown precipitate demonstrating a strong increase in $\mathrm{H}_{2} \mathrm{O}_{2}$ release by $\triangle P a A t p e$ and $\triangle P a A t p g$ cultures (Fig. 3C).

In order to link these different age-dependent changes to mitochondrial functions, we measured respiration of $P$. anserina deletion strains. Clearly, total oxygen consumption of deletion strains is reduced in comparison to the wild type (Fig. 3D). Furthermore, we determined respira- tion of wild-type, $\triangle P a A t p e$ and $\triangle P a A t p g$ strains in the presence and absence of respiration inhibitors potassium cyanide (KCN), inhibiting complex IV (COX), and salicylhydroxamic acid (SHAM), inhibiting the alternative oxidase AOX (Fig. 3E). Notably, in the two deletion strains alternative respiration is slightly increased as compensatory response to OXPHOS impairments and increased mitochondrial stress [46-48]. These data are in concordance with studies in yeast in which mutations of several subunits of $\mathrm{F}_{1} \mathrm{~F}_{\mathrm{o}}$-ATP-synthase like Atp4 [49] or Su e and Su g [50, 51] lead to a destabilization of of the complex and a reduced cOX activity. 
A

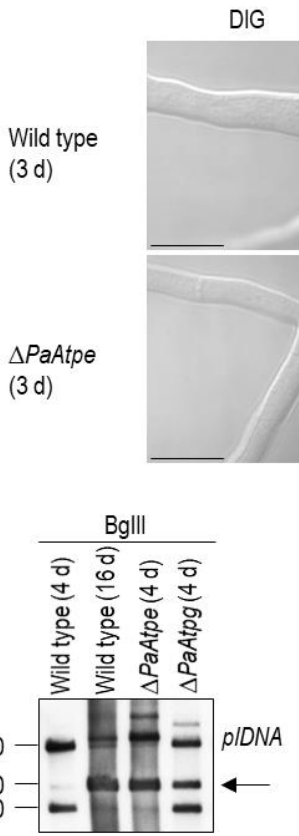

E

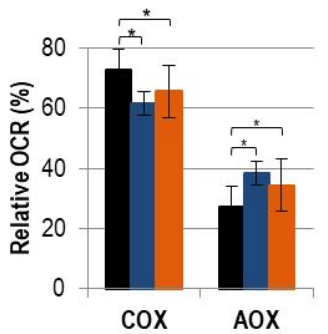

H

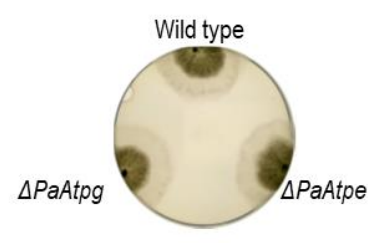

C

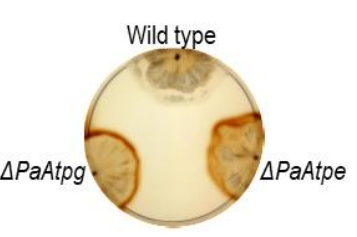

$\mathbf{F}$

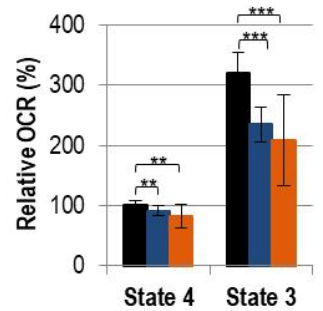

I

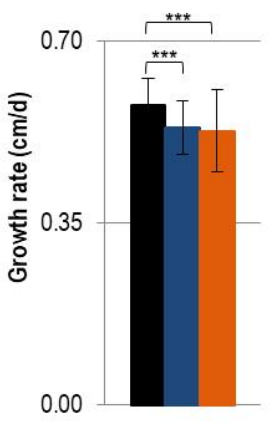

G
DIG MTRed
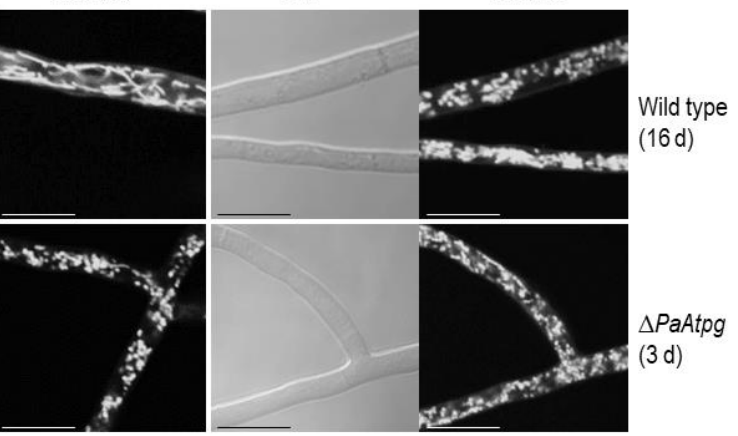
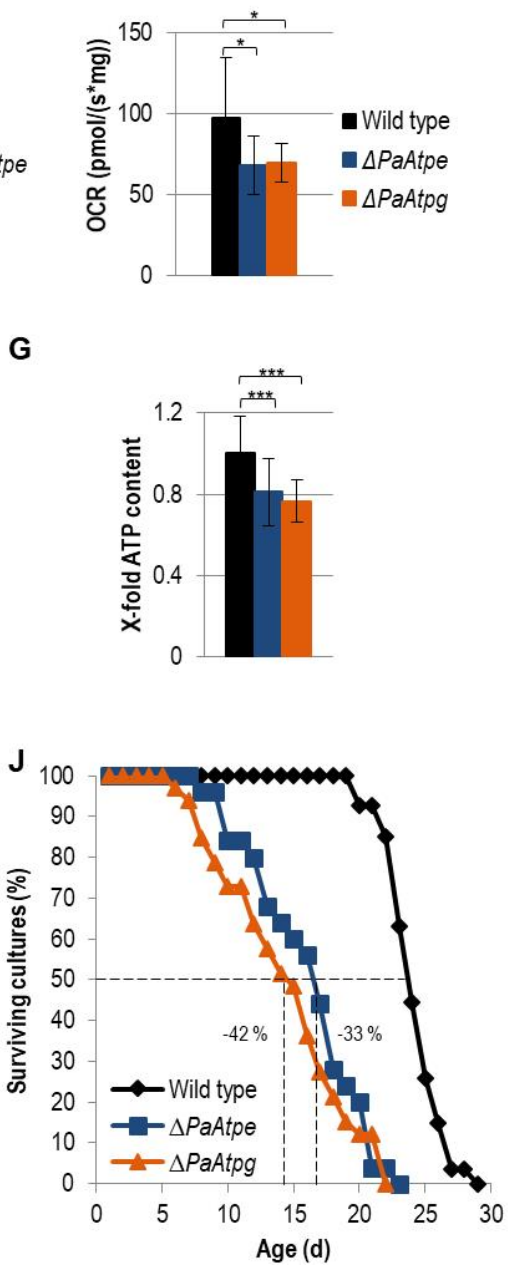

FIGURE 3: Impairments of mitochondrial functions affect growth and lifespan in deletion mutants. (A) Comparison of mitochondrial morphology of 3-days and 16-days old wild-type strains with those of $\triangle$ PaAtpe and $\triangle$ PaAtpg (3 d) by fluorescence microscopy. Mitochondria were stained with MitoTracker ${ }^{\circledast}$ Red and analyzed by confocal fluorescence microscopy. Scale bars: $10 \mu \mathrm{m}$. (B) Representative Southern blot analysis of Bglll-digested gDNA of wild-type (4-days and 16-days old), $\triangle P a A t p e$ and $\triangle P a A t p g$ strains (4-days old). The 2500 bp age-specific plDNA (arrow) fragment was detected with a specific pIDNA probe. (C) Release of ROS species $\mathrm{H}_{2} \mathrm{O}_{2}$ from wild-type (6-days $\mathrm{n}=3$ ), $\triangle P a A t p e(6$-days $\mathrm{n}=3$ ) and $\triangle P a A t p g$ strains (6-days $\mathrm{n}$ = 3) visualized by DAB precipitation. (D) Oxygen consumption (basal respiration) of 6-days old deletion mutant mycelium compared to respiration of 6-days old wild type mycelium ( $n=3-4$ biological replicates with a total number of 12-16 technical replicates). (E) Relative COX- and AOX-dependent oxygen consumption rate (OCR) of 6-days old wild-type and 6-days old deletion strains mycelium after treatment with KCN (COX-inhibitor) or SHAM (AOX-inhibitor). (F) Relative complex I-dependent OCR of 6-days old wild-type mitochondria compared to mitochondria from 6-days old deletion mutants ( $n=4-8$ biological replicates with a total number of 15-28 technical replicates). State 4 OCR of mitochondria from wild type was set to $100 \%$. (G) ATP production of 6-days old wild-type and 6-days old deletion mutant mitochondria after removal of samples during state 3 respiration measurements. Samples of three biological replicates and two technical replicates, respectively, were measured three times by a luminescence-based assay. (H) Phenotypic analysis of indicated strains growing on $\mathrm{M} 2$ medium for 4 days. (I) Growth rate of wild type $(0.58 \pm 0.05 \mathrm{~cm} / \mathrm{d}, \mathrm{n}=27), \Delta$ PaAtpe $(0.53 \pm 0.05 \mathrm{~cm} / \mathrm{d}, \mathrm{n}=25)$ and $\triangle P a A t p g(0.53 \pm 0.08 \mathrm{~cm} / \mathrm{d}, \mathrm{n}=33)$ growing on $\mathrm{M} 2$ medium. Growth rate was determined from day 3 to 6 of growth. (J) Lifespan analysis of wild type (mean lifespan $=23.3 \mathrm{~d}, \mathrm{n}=27$ ), $\triangle$ PaATPe (mean lifespan $=15.1 \mathrm{~d}, \mathrm{n}=25$, ) and $\triangle P a A T P g($ mean lifespan $=13.5 \mathrm{~d}, \mathrm{n}=$ 33) growing on $M 2$ medium. The error bars represent the standard deviation and the P-values were determined by Student's t test. 
Determination of the relative oxygen consumption rate (OCR) via complex I of isolated mitochondria from wildtype, $\triangle P a A t p e$ and $\triangle P a A t p g$ strains revealed that state 4 (ADP-independent) as well as state 3 (ADP-dependent) respiration is significantly decreased in deletion mutants. State 3 respiration is most strongly affected (Fig. 3F). An impaired respiration often results in an inefficient ATP production. To test $F_{1} F_{0}$-ATP-synthase activity, we removed part of the samples used for state 3 respiration measurements and determined ATP production in this state using a luciferin-luciferase assay. We found that ATP content in deletion strains is significantly reduced compared to the wild type (Fig. 3G) indicating that monomeric $\mathrm{F}_{1} \mathrm{~F}_{\mathrm{o}}$-ATPsynthase is not as efficient as the dimeric form. Thus, the dimerization of $F_{1} F_{0}$-ATP-synthase is not only important for $\mathrm{F}_{1} \mathrm{~F}_{\mathrm{o}}$-ATP-synthase dimerization and mitochondrial ultrastructure but also for energy transduction. Similar effects were also observed in yeast and HeLa cells. In HeLa cells, downregulation of dimer-specific genes leads to an increase of doubling-times and a reduction of OXPHOS capacity [33]. Yeast cells in which either one of the two dimer assembly factors is ablated display an abnormal mitochondrial morphology, a decreased growth rate on nonfermentable carbon sources and reduced respiration [12, 30].

The effect of reduced ATP generation in PaAtpe and PaAtpg deletion strains is not apparent in the first days (e.g. 4 days) of growth. At this time, cultures display the same morphology and radial growth rate as the wild type (Fig. $3 \mathrm{H}$ ). Later on (days $3-6$ ), growth rate of deletion strains becomes slightly, but significantly reduced when compared to the wild type (Fig. 3I). The reduction of the growth rate in the presenescent life phase is another aging marker that together with the described changes in mitochondrial morphology and ultrastructure as well as mitochondria dysfunction, reorganization of mtDNA and the increase in ROS production suggests an acceleration of the aging process in $\triangle P a A t p e$ and $\triangle P a A t p g$ strains. This conclusion was finally verified by determination of the lifespan of the two deletion strains. In comparison to the wild type, the lifespan of the mutants is strongly reduced by $33 \%$ and $42 \%$, respectively (Fig. 3J). Until now, such an effect on biological aging was not reported in any of the different established aging models, including yeast in which $F_{1} F_{0}-$ ATP-synthase dimerization factors were extensively studied.

\section{Conclusions and perspectives}

Here we provide novel data on the identification of individual components involved in the control of mitochondrial inner membrane remodeling and their impact on biological aging. These experimental data extend a model that was proposed from earlier cryo-electron microscopy studies [25] and from data of genetic interventions into the induction of PCD via the overexpression of a gene coding for cyclophilin $D$, a regulator of the mitochondrial permeability transition (mPT) [29]. In this particular strain, it was demonstrated that autophagy is induced to an extent that leads to autophagic cell death [52]. Significantly, CYPD is known to interact with the lateral stalk of the $F_{1} F_{0}$-ATPsynthase decreasing its activity [53]. Moreover, $F_{1} F_{0}-A T P-$ synthase dimers were demonstrated to be part of the mPTP [54] and that opening of the MPTP correlates with the dissociation of dimers [55]. Overall, these and the data presented in our current study highlight the central role of $\mathrm{F}_{1} \mathrm{~F}_{\mathrm{o}}$-ATP-synthase in mitochondrial biology. However, the regulatory circuits and the mechanisms involved in the different aspects of $F_{1} F_{0}$-ATP-synthase function remain to be unraveled in detail. Concerning the role in mitochondrial ultrastructure remodeling, the identification and the characterization of components involved in this process is crucial. Apart from the role of $F_{1} F_{0}$-ATP-synthase dimers at the cristae tips, the MICOS complex has been recently identified [18-20]. Interestingly, it was found that both complexes are necessary for cristae formation. Notwithstanding that the core MICOS protein Mic60 and dimer assembly factors Su e and Su g play antagonistic roles [14], the other core MICOS protein, Mic10, was found to interact with $\mathrm{Su}$ e of the $\mathrm{F}_{1} \mathrm{~F}_{\mathrm{o}}$-ATP-synthase $[21,22]$. Furthermore, the demonstration of a $\mathrm{F}_{1} \mathrm{~F}_{\mathrm{o}}$-ATP-synthase oligomerization promoting effect of Mic27 [21] also suggests an interplay of the MICOS complex with the $\mathrm{F}_{1} \mathrm{~F}_{\mathrm{o}}$-ATP-synthase dimer. The elucidation of regulation of these crosstalks will certainly be a key aspect of future investigations.

\section{MATERIALS AND METHODS}

\section{$P$. anserina strains and cultivation}

In this study wild type ' $s$ ' [56] and the newly generated deletion strains $\triangle P a A t p e$ and $\triangle P a A t p g$ were used. Strains were grown on standard cornmeal agar (BMM) at $27^{\circ} \mathrm{C}$ under constant light [57]. For spore germination, standard cornmeal agar (BMM) supplemented with $60 \mathrm{mM}$ ammonium acetate was used and incubated at $27^{\circ} \mathrm{C}$ in dark for 2 days. For all experiments strains were cultivated on $\mathrm{M} 2$ plates for 2 days at $27^{\circ} \mathrm{C}$ under constant light and transferred in CM liquid medium for 2 days at $27^{\circ} \mathrm{C}$ under constant light. Flasks were shaken at 160 rounds per minute (rpm). All strains used in this study were derived from monokaryotic ascospores [57].

\section{Cloning procedures and generation of $\boldsymbol{P}$. anserina mutants} Deletion of PaAtpe and PaAtpg was performed with the method described by Hamann et al., 2005 [38]. Briefly, a small 5'-flank of genes PaAtpe and PaAtpg, respectively, was amplified with oligonucleotides ATPe_KO1 (5'GTGGTACCCGCTGTGAGAGCTTCTTC- $\left.3^{\prime}\right)$, ATPe_KO2 (5' GCAAGCTTTTTGAGGAATCTGGGGGC-3'), ATPg_KO1 (5'CGGGTACCGTGAAGAGCGTATGTTGG-3') and ATPg_KO2 (5'GCAAGCTTTTCGAACTTCGGGCACTG-3'). A small $3^{\prime}$-flank of the same genes was amplified with oligonucleotides ATPe_KO3 (5'- GCACTAGTACTGTCCGTCGACGAACT-3'), ATPe_KO4 (5'AAAGCGGCCGCTCAACGATGTGACATTG-3'), ATPg_KO3 (5'CTACTAGTTCTTAGCGCAGGGAGGTG-3') and ATPg_KO4 (5'CAGCGGCCGCAGGTTGCAACAGTAGTAG-3'). Recognition sites of restriction endonucleases are underlined. The $5^{\prime}$ fragments were digested with $\mathrm{Kpnl}$ and HindIII and the $3^{\prime}$ fragments with Bcul and Notl and ligated into previously digested plasmid pKO4. The resulting plasmids were termed pAtpeKO1 and pAtpgKO1 and contain a resistance cassette with phleomycin and blasticidin marker genes for fungal and bacterial selection, 
respectively. The flanked resistance cassettes were digested with Notl and Kpnl and used for transformation of Escherichia coli strain KS272 bearing the plasmid pKOBEG [58] and the cosmid 15C5 containing the PaAtpe locus or cosmid 13B10 containing the PaAtpg locus, respectively. Homologous recombination between the flanks of the resistance cassette and cosmid $15 \mathrm{C} 5$ and $13 \mathrm{~B} 10$ leads to generation of $\triangle \mathrm{Atpe} 15 \mathrm{C} 5$ and $\Delta$ Atpg13B10, which contain the two resistance markers surrounded by large flanks. The cosmid was isolated and used for transformation of wild type $P$. anserina. Selection of positive PaAtpe and PaAtpg deletion strains was performed by growth on medium containing phleomycin. The deletion of PaAtpe and PaAtpg was verified by Southern blot analysis as described below.

\section{Southern blot analysis}

Total DNA was isolated according to Lecellier and Silar, 1994 [59]. DNA restriction, gel electrophoresis and Southern blotting were performed according to standard protocols. For Southern blot hybridization and detection, digoxigenin-labeled hybridization probes ('DIG DNA Labeling and Detection Kit') were used according to the manufacturer's instructions. The PaAtpe-specific hybridization probe was amplified by PCR using the oligonucleotides Pa_1_3740-1 (5' AATGGCCTCTTCCGGAGTC-3') and Pa_1_3740-2 (5'CTCGAGGTCGAAGCTTGAG-3') corresponding to 511 nucleotides of the gene PaAtpe. The PaAtpg-specific hybridization probe was amplified by $P C R$ using the oligonucleotides Pa_1_2480-3 (5'- TCTTTCGCCCTCCTCTCAC-3') and Pa_1_24804 (5'- AGACGATCTTGGCCACCTC- $3^{\prime}$ ) corresponding to $418 \mathrm{nu}$ cleotides of the gene PaAtpg. As a hybridization probe specific for the phleomycin resistance gene (Ble), the $1293 \mathrm{bp} \mathrm{BamHI-}$ fragment of the plasmid pKO4 [60] was used. As a hybridization probe specific for the pIDNA the $2500 \mathrm{bp} \mathrm{BamHI-fragment}$ of the plasmid pSP17 [61] was used.

\section{Growth rate and lifespan determination}

To determine lifespan and growth rate of $P$. anserina strains used in this study, monokaryotic ascospores were isolated from crosses of respective strains and germinated for 2 days at $27^{\circ} \mathrm{C}$ in the dark on BMM supplemented with $60 \mathrm{mM}$ ammonium acetate. After germination mycelia were placed on $\mathrm{M} 2$ medium [57] and incubated at $27^{\circ} \mathrm{C}$ under constant light. The lifespan is defined as the period of linear growth in days (d). The growth rate was measured as growth in centimeters per day $(\mathrm{cm} / \mathrm{d})$.

\section{Transcript analysis}

Plates containing M2 medium covered with cellophane were inoculated with small pieces of mycelium and incubated for 2 days at $27^{\circ} \mathrm{C}$ under constant light. Total RNA was isolated from scraped fungal mycelium using the Nucleo Spin ${ }^{\circledR}$ RNA Plant-Kit (Macherey-Nagel). Reverse transcription of $1 \mathrm{mg}$ total RNA was performed with the RevertAid ${ }^{\mathrm{TM}} \mathrm{M}$-MuLV reverse transcriptase (ThermoScientific) according to the manufacturer's instruction. Real-time PCR was realized using iQ SYBR Green Supermix (BioRad) followed by the manufacturer's protocol. For each gene, the efficiency $(E)$ of the primer pairs was calculated based on a real-time PCR with a dilution series of CDNA according to $E=10^{[-1 / \text { Slope] }}$ [62]. The relative expression level (normalized to the level of the porin transcript) was calculated according to the following formula: Relative expression=
$\left(\mathrm{E}(\text { porin })^{\wedge} \mathrm{CP}(\right.$ porin $\left.)\right) /\left(\mathrm{E}(\text { target gene })^{\wedge} \mathrm{CP}(\right.$ target gene $\left.)\right)$ with: $\mathrm{E}=$ $\mathrm{PCR}$ efficiency of the respective primer pair and $\mathrm{CP}=$ crossing point for each transcript.

\section{Mitochondria isolation}

Mitochondria of $P$. anserina cultures were isolated by differential centrifugation for measurement of mitochondrial oxygen consumption and BN-PAGE analysis [57].

\section{Blue-native polyacrylamide gels (BN-PAGE)}

BN-PAGE was performed as previously described [63]. For each sample $75-100 \mu \mathrm{g}$ of mitochondrial protein extracts were solubilized using a digitonin/protein ratio of 3:1 (w/w). Linear gradient gels (4-13\%) overlaid with $3.5 \%$ stacking gels were used for separation of solubilized mitochondria. Respiratory chain components were subsequently visualized by Coomassie blue staining and assigned as described [64].

\section{Mitochondrial oxygen consumption}

Measurement of mitochondrial oxygen consumption was performed at $27^{\circ} \mathrm{C}$ using two different high-resolution respirometers (Oxygraph-2k series C and G, Oroboros Instruments, Innsbruck, Austria). $200 \mu \mathrm{g}$ freshly prepared mitochondria were injected into $2 \mathrm{ml}$ air saturated oxygen buffer $(0.3 \mathrm{M}$ sucrose, $10 \mathrm{mM} \mathrm{KH}_{2} \mathrm{PO}_{4}, 5 \mathrm{mM} \mathrm{MgCl}$, $1 \mathrm{mM}$ EDTA, $10 \mathrm{mM} \mathrm{KCl}, 0.1 \%$ $\mathrm{BSA} ; \mathrm{pH}=7.2$ ). To determine the complex I-dependent state 4 respiration (state 4) $20 \mathrm{mM}$ pyruvate and $5 \mathrm{mM}$ malate were added. Thereafter, $1.5 \mathrm{mM}$ ADP was added to determine complex I-dependent state 3 respiration (state 3 ).

Determination of complex IV- and AOX-dependent oxygen consumption was performed using strains cultivated on $\mathrm{M} 2$ medium for 2 days and in $\mathrm{CM}$ liquid medium for 2 days as described above. Small pieces of mycelium were subsequently transferred into the respirometer and oxygen consumption was measured in liquid $\mathrm{CM}$ medium according to the manufacturer's instructions. $1 \mathrm{mM} \mathrm{KCN}$ was added to inhibit respiration via complex IV. $4 \mathrm{mM}$ salicylhydroxamic acid (SHAM) was added to inhibit respiration via AOX.

Data were analyzed using the manufacturer's software DatLab 6.

\section{ATP measurement}

For determination of ATP production samples were removed after measurement of state 3 respiration. Thereafter samples were boiled in a water bath for 15 minutes. After boiling they were centrifuged for $10 \mathrm{~min}$ and 14,000 rpm and transferred into new reaction tubes. The supernatant was diluted (1:30) and ATP amount was measured by using the ATP Bioluminescence Assay Kit (CLS II, Roche) adapted for use in a microtiter plate format. The assay was performed according to the manufacturer's instructions.

\section{Fluorescence microscopy}

$P$. anserina mycelia were grown on microscope slides with a central depression that is filled with $\mathrm{M} 2$ agar medium and incubated for $1-2$ days at $27^{\circ} \mathrm{C}$ and constant light. Hyphae were stained with $1 \mu \mathrm{M}$ of the mitochondrial specific dye Mito Tracker CMX ROS (Invitrogen) and visualized using a confocal laser scanning microscope (CLSM TCS SP5, Leica). 


\section{Electron microscopy}

Electron microscopy of chemically fixed isolated mitochondria was performed as described [65].

\section{Hydrogen peroxide release assay}

To determine the $\mathrm{H}_{2} \mathrm{O}_{2}$ release mycelia were cultivated on $\mathrm{M} 2$ medium at $27^{\circ} \mathrm{C}$ in the dark for 4 days. The plates were flooded with a solution containing $100 \mathrm{mM}$ Tris/ $\mathrm{HCl} \mathrm{pH} 6.9$ and 2.5 $\mathrm{mM}$ diaminobenzidine (DAB) and incubated for $30 \mathrm{~min}$ in the dark and $27^{\circ} \mathrm{C}$. Subsequently, the solution was poured off and the plates were incubated again for $3 \mathrm{~h}$ at $27^{\circ} \mathrm{C}$ in the dark.

\section{Statistical analysis}

All statistical significances were calculated using the Student ttest. P-values $<0.05$ were considered statistically significant. $\mathrm{P}$ $\leq 0.05:^{*}, \mathrm{P} \leq 0.01:^{* *}, \mathrm{P} \leq 0.001$ : $^{* *}$.

\section{ACKNOWLEDGEMENTS}

This work was supported by grants of the Deutsche Forschungsgemeinschaft (DFG: Os75/17-1) to HDO and via the Cluster of Excellence 'Macromolecular Complexes'. We thank Alexandra Werner for preparation of $P$. anserina mitochondria of defined age.

\section{REFERENCES}

1. Zick M, Rabl R, Reichert AS (2009). Cristae formation-linking ultrastructure and function of mitochondria. Biochim Biophys Acta 1793(1): 5-19. doi: 10.1016/j.bbamcr.2008.06.013

2. Cogliati S, Enriquez JA, Scorrano L (2016). Mitochondrial cristae: where beauty meets functionality. Trends Biochem Sci 41(3): 261-273. doi: 10.1016/j.tibs.2016.01.001

3. Harner ME, Unger AK, Geerts WJ, Mari M, Izawa $T$, Stenger M, Geimer S, Reggiori F, Westermann B, Neupert W (2016). An evidence based hypothesis on the existence of two pathways of mitochondrial crista formation. Elife 5: e18853. doi: 10.7554/eLife. 18853

4. Rampelt $\mathrm{H}$, Zerbes RM, van der Laan M, Pfanner $\mathrm{N}$ (2017). Role of the mitochondrial contact site and cristae organizing system in membrane architecture and dynamics. Biochim Biophys Acta 1864(4): 737-746. doi: 10.1016/j.bbamcr.2016.05.020

5. Jonckheere Al, Smeitink JA, Rodenburg RJ (2012). Mitochondrial ATP synthase: architecture, function and pathology. J Inherit Metab Dis 35(2): 211-225. doi: 10.1007/s10545-0119382-9

6. Tatsuta T, Langer T (2008). Quality control of mitochondria: protection against neurodegeneration and ageing. EMBO J 27(2): 306-314. doi: 10.1038/sj.emboj.7601972

7. Coskun P, Wyrembak J, Schriner SE, Chen HW, Marciniack C, Laferla F, Wallace DC (2012). A mitochondrial etiology of Alzheimer and Parkinson disease. Biochim Biophys Acta 1820(5): 553-564. doi: 10.1016/j.bbagen.2011.08.008

8. Itoh K, Nakamura K, lijima M, Sesaki H (2013). Mitochondrial dynamics in neurodegeneration. Trends Cell Biol 23(2): 6471. doi: 10.1016/j.tcb.2012.10.006

\section{AUTHOR CONTRIBUTION}

HDO conceived and supervised this study. MS performed electron microscopy and, together with BW, analyzed the data. NGR performed the rest of the experiments and NGR and HDO analyzed data. HDO and NGR wrote the manuscript. BW and MS edited the manuscript. All authors read and approved the final manuscript.

\section{CONFLICT OF INTEREST}

The authors declare that they have no conflict of interest.

\section{COPYRIGHT}

(C) 2018 Rampello et al. This is an open-access article released under the terms of the Creative Commons Attribution (CC BY) license, which allows the unrestricted use, distribution, and reproduction in any medium, provided the original author and source are acknowledged.

Please cite this article as: Nadia G Rampello, Maria Stenger, Benedikt Westermann, Heinz D Osiewacz (2018). Impact of F1Fo-ATPsynthase dimer assembly factors on mitochondrial function and organismic aging. Microbial Cell 5(4): 198-207. doi: 10.15698/mic2018.04.625

9. Lin MT, Beal MF (2006). Mitochondrial dysfunction and oxidative stress in neurodegenerative diseases. Nature 443(7113): 787-795. doi: 10.1038/nature05292

10. Baker BM, Haynes CM (2011). Mitochondrial protein quality control during biogenesis and aging. Trends Biochem Sci 36(5): 254-261. doi: 10.1016/j.tibs.2011.01.004

11. Osiewacz HD, Bernhardt D (2013). Mitochondrial quality control: impact on aging and life span - a mini-review. Gerontology 59(5): 413-420. doi: 10.1159/000348662

12. Bornhövd C, Vogel F, Neupert W, Reichert AS (2006). Mitochondrial membrane potential is dependent on the oligomeric state of F1Fo -ATP synthase supracomplexes. J Biol Chem 281(20): 13990-13998. doi: 10.1074/jbc.M512334200

13. Strauss M, Hofhaus G, Schroder RR, Kühlbrandt W (2008). Dimer ribbons of ATP synthase shape the inner mitochondrial membrane. EMBO J 27(7): 1154-1160. doi: 10.1038/emboj.2008.35

14. Rabl R, Soubannier V, Scholz R, Vogel F, Mendl N, VasiljevNeumeyer A, Korner C, Jagasia R, Keil T, Baumeister W, Cyrklaff M, Neupert W, Reichert AS (2009). Formation of cristae and crista junctions in mitochondria depends on antagonism between Fcj1 and Su e/g. J Cell Biol 185(6): 1047-1063. doi: $10.1083 / j c b .200811099$

15. Davies KM, Strauss M, Daum B, Kief JH, Osiewacz HD, Rycovska A, Zickermann V, Kühlbrandt W (2011). Macromolecular organization of ATP synthase and complex I in whole mitochondria. Proc Natl Acad Sci U S A 108(34): 14121-14126. doi: 10.1073/pnas.1103621108

16. Davies KM, Anselmi C, Wittig I, Faraldo-Gomez JD, Kühlbrandt W (2012). Structure of the yeast F1Fo-ATP syn- 
thase dimer and its role in shaping the mitochondrial cristae. Proc Natl Acad Sci U S A 109(34): 13602-13607. doi: 10.1073/pnas.1204593109

17. Pfanner N, van der Laan M, Amati P, Capaldi RA, Caudy AA, Chacinska A, Darshi M, Deckers M, Hoppins S, Icho T, Jakobs S, Ji J, Kozjak-Pavlovic V, Meisinger C, Odgren PR, Park SK, Rehling P, Reichert AS, Sheikh MS, Taylor SS, Tsuchida N, van der Bliek AM, van der Klei IJ, Weissman JS, Westermann B, Zha J, Neupert W, Nunnari J (2014). Uniform nomenclature for the mitochondrial contact site and cristae organizing system. J Cell Biol 204(7): 1083-1086. doi: 10.1083/jcb.201401006

18. Harner M, Korner C, Walther D, Mokranjac D, Kaesmacher J, Welsch U, Griffith J, Mann M, Reggiori F, Neupert W (2011). The mitochondrial contact site complex, a determinant of mitochondrial architecture. EMBO J 30(21): 4356-4370. doi: 10.1038/emboj.2011.379

19. Hoppins S, Collins SR, Cassidy-Stone A, Hummel E, Devay RM, Lackner LL, Westermann B, Schuldiner M, Weissman JS, Nunnari J (2011). A mitochondrial-focused genetic interaction map reveals a scaffold-like complex required for inner membrane organization in mitochondria. J Cell Biol 195(2): 323340. doi: 10.1083/jcb.201107053

20. von der Malsburg K, Muller JM, Bohnert M, Oeljeklaus S, Kwiatkowska P, Becker T, Loniewska-Lwowska A, Wiese S, Rao $S$, Milenkovic D, Hutu DP, Zerbes RM, Schulze-Specking A, Meyer HE, Martinou JC, Rospert S, Rehling P, Meisinger C, Veenhuis M, Warscheid B, van der Klei IJ, Pfanner N, Chacinska $A$, van der Laan $M$ (2011). Dual role of mitofilin in mitochondrial membrane organization and protein biogenesis. Dev Cell 21(4): 694-707. doi: 10.1016/j.devcel.2011.08.026

21. Eydt K, Davies KM, Behrendt C, Wittig I, Reichert AS (2017). Cristae architecture is determined by an interplay of the MICOS complex and the F1Fo ATP synthase via Mic27 and Mic10. Microb Cell 4(8): 259-272. doi: 10.15698/mic2017.08.585

22. Rampelt $H$, Bohnert $M$, Zerbes RM, Horvath SE, Warscheid B, Pfanner N, van der Laan M (2017). Mic10, a core subunit of the mitochondrial contact site and cristae organizing system, interacts with the dimeric F1Fo-ATP synthase. J Mol Biol 429(8): 1162-1170. doi: 10.1016/j.jmb.2017.03.006

23. Mannella CA (2006). Structure and dynamics of the mitochondrial inner membrane cristae. Biochim Biophys Acta 1763(5-6): 542-548. doi: 10.1016/j.bbamcr.2006.04.006

24. Wai T, Langer T (2016). Mitochondrial dynamics and metabolic regulation. Trends Endocrinol Metab 27(2): 105-117. doi: 10.1016/j.tem.2015.12.001

25. Daum B, Walter A, Horst A, Osiewacz HD, Kühlbrandt W (2013). Age-dependent dissociation of ATP synthase dimers and loss of inner-membrane cristae in mitochondria. Proc Natl Acad Sci U S A 110(38): 15301-15306. doi: 10.1073/pnas.1305462110

26. Hamann A, Brust D, Osiewacz HD (2007). Deletion of putative apoptosis factors leads to lifespan extension in the fungal ageing model Podospora anserina. Mol Microbiol 65(4): 948958. doi: 10.1111/j.1365-2958.2007.05839.x
27. Hamann A, Brust D, Osiewacz HD (2008). Apoptosis pathways in fungal growth, development and ageing. Trends Microbiol 16(6): 276-283. doi: 10.1016/j.tim.2008.03.003

28. Baines CP (2009). The molecular composition of the mitochondrial permeability transition pore. J Mol Cell Cardiol 46(6): 850-857. doi: 10.1016/j.yjmcc.2009.02.007

29. Brust D, Daum B, Breunig C, Hamann A, Kühlbrandt W, Osiewacz HD (2010). Cyclophilin D links programmed cell death and organismal aging in Podospora anserina. Aging Cell 9(5): 761-775. doi: 10.1111/j.1474-9726.2010.00609.x

30. Arnold I, Pfeiffer K, Neupert W, Stuart RA, Schägger $H$ (1998). Yeast mitochondrial F1Fo -ATP synthase exists as a dimer: identification of three dimer-specific subunits. EMBO J 17(24): 7170-7178. doi: 10.1093/emboj/17.24.7170

31. Wagner K, Rehling P, Sanjuan Szklarz LK, Taylor RD, Pfanner $N$, van der Laan M (2009). Mitochondrial F1Fo-ATP synthase: the small subunits $\mathrm{e}$ and $\mathrm{g}$ associate with monomeric complexes to trigger dimerization. J Mol Biol 392(4): 855-861. doi: 10.1016/j.jmb.2009.07.059

32. Paumard P, Vaillier J, Coulary B, Schaeffer J, Soubannier V, Mueller DM, Brethes D, di Rago JP, Velours J (2002). The ATP synthase is involved in generating mitochondrial cristae morphology. EMBO J 21(3): 221-230. doi 10.1093/emboj/21.3.221

33. Habersetzer J, Larrieu I, Priault M, Salin B, Rossignol R, Brethes D, Paumard P (2013). Human F1FO ATP synthase, mitochondrial ultrastructure and OXPHOS impairment: a (super)complex matter? PLoS One 8(10): e75429. doi: 10.1371/journal.pone.0075429

34. Philipp O, Hamann A, Servos J, Werner A, Koch I, Osiewacz HD (2013). A genome-wide longitudinal transcriptome analysis of the aging model Podospora anserina. PLoS One 8(12): e83109. doi: 10.1371/journal.pone.0083109

35. Knuppertz L, Hamann A, Pampaloni F, Stelzer E, Osiewacz HD (2014). Identification of autophagy as a longevityassurance mechanism in the aging model Podospora anserina. Autophagy 10(5): 822-834. doi: 10.4161/auto.28148

36. Wagner K, Perschil I, Fichter CD, van der Laan M (2010). Stepwise assembly of dimeric F(1)F(o)-ATP synthase in mitochondria involves the small $F(0)$-subunits $k$ and $i$. Mol Biol Cell 21(9): 1494-1504. doi: 10.1091/mbc.E09-12-1023

37. Everard-Gigot V, Dunn CD, Dolan BM, Brunner S, Jensen RE, Stuart RA (2005). Functional analysis of subunit e of the F1Fo-ATP synthase of the yeast Saccharomyces cerevisiae: importance of the N-terminal membrane anchor region. Eukaryot Cell 4(2): 346-355. doi: 10.1128/EC.4.2.346-355.2005

38. Hamann A, Krause K, Werner A, Osiewacz HD (2005). A two-step protocol for efficient deletion of genes in the filamentous ascomycete Podospora anserina. Curr Genet 48(4): 270-275. doi: 10.1007/s00294-005-0018-1

39. Scheckhuber CQ, Erjavec N, Tinazli A, Hamann A, Nystrom $\mathrm{T}$, Osiewacz HD (2007). Reducing mitochondrial fission results in increased life span and fitness of two fungal ageing models. Nat Cell Biol 9(1): 99-105. doi: 10.1038/ncb1524 
40. Stahl U, Lemke PA, Tudzynski P, Kück U, Esser K (1978). Evidence for plasmid like DNA in a filamentous fungus, the ascomycete Podospora anserina. Mol Gen Genet 162(3): 341343. PMID: 683172

41. Cummings DJ, Belcour L, Grandchamp C (1979). Mitochondrial DNA from Podospora anserina. I. Isolation and characterization. Mol Gen Genet 171(3): 229-238. PMID: 286867

42. Belcour L, Begel O, Mosse MO, Vierny C (1981). Mitochondrial DNA amplification in senescent cultures of Podospora anserina: variability between the retained, amplified sequences. Curr Genet 3(1): 13-21. doi: 10.1007/BF00419575

43. Kück U, Stahl U, Esser K (1981). Plasmid-like DNA is part of mitochondrial DNA in Podospora anserina. Curr Genet 3(2): 151-156. doi: 10.1007/BF00365719

44. Osiewacz HD, Esser K (1984). The mitochondrial plasmid of Podospora anserina: a mobile intron of a mitochondrial gene. Curr Genet 8(4): 299-305. doi: 10.1007/BF00419728

45. Wiemer M, Osiewacz HD (2014). Effect of paraquatinduced oxidative stress on gene expression and aging of the filamentous ascomycete Podospora anserina. Microb Cell 1(7): 225-240. doi: 10.15698/mic2014.07.155

46. Borghouts C, Werner A, Elthon T, Osiewacz HD (2001). Copper-modulated gene expression and senescence in the filamentous fungus Podospora anserina. Mol Cell Biol 21(2): 390-399. doi: 10.1128/MCB.21.2.390-399.2001

47. Lorin S, Dufour E, Boulay J, Begel O, Marsy S, SainsardChanet A (2001). Overexpression of the alternative oxidase restores senescence and fertility in a long-lived respirationdeficient mutant of Podospora anserina. Mol Microbiol 42(5): 1259-1267. doi: 10.1046/j.1365-2958.2001.02690.x

48. Scheckhuber CQ, Houthoofd K, Weil AC, Werner A, De Vreese A, Vanfleteren JR, Osiewacz HD (2011). Alternative oxidase dependent respiration leads to an increased mitochondrial content in two long-lived mutants of the aging model Podospora anserina. PLoS One 6(1): e16620. doi: 10.1371/journal.pone.0016620

49. Paul MF, Velours J, Arselin de Chateaubodeau G, Aigle M, Guerin B (1989). The role of subunit 4, a nuclear-encoded protein of the F0 sector of yeast mitochondrial ATP synthase, in the assembly of the whole complex. Eur J Biochem 185(1): 163-171. doi: 10.1111/j.1432-1033.1989.tb15098.x

50. Boyle GM, Roucou X, Nagley P, Devenish RJ, Prescott M (1999). Identification of subunit $g$ of yeast mitochondrial F1FOATP synthase, a protein required for maximal activity of cytochrome c oxidase. Eur J Biochem 262(2): 315-323. doi: 10.1046/j.1432-1327.1999.00345.x

51. Saddar S, Dienhart MK, Stuart RA (2008). The F1FO-ATP synthase complex influences the assembly state of the cytochrome bc1-cytochrome oxidase supercomplex and its association with the TIM23 machinery. J Biol Chem 283(11): 66776686. doi: 10.1074/jbc.M708440200

52. Kramer P, Jung AT, Hamann A, Osiewacz HD (2016). Cyclophilin $D$ is involved in the regulation of autophagy and affects the lifespan of $P$. anserina in response to mitochondrial oxida- tive stress. Front Genet 7: 165. doi: 10.3389/fgene.2016.00165

53. Giorgio V, Bisetto E, Soriano ME, Dabbeni-Sala F, Basso E, Petronilli V, Forte MA, Bernardi P, Lippe G (2009). Cyclophilin $D$ modulates mitochondrial FoF1-ATP synthase by interacting with the lateral stalk of the complex. J Biol Chem 284(49): 33982-33988. doi: 10.1074/jbc.M109.020115

54. Giorgio V, von Stockum S, Antoniel M, Fabbro A, Fogolari F, Forte M, Glick GD, Petronilli V, Zoratti M, Szabo I, Lippe G, Bernardi $P$ (2013). Dimers of mitochondrial ATP synthase form the permeability transition pore. Proc Natl Acad Sci U S A 110(15): 5887-5892. doi: 10.1073/pnas.1217823110

55. Bonora M, Morganti C, Morciano G, Pedriali G, Lebiedzinska-Arciszewska M, Aquila G, Giorgi C, Rizzo P, Campo G, Ferrari R, Kroemer G, Wieckowski MR, Galluzzi L, Pinton P (2017). Mitochondrial permeability transition involves dissociation of F1Fo ATP synthase dimers and C-ring conformation. EMBO Rep 18(7): 1077-1089. doi: 10.15252/embr.201643602

56. Rizet G (1953). Impossibility of obtaining uninterrupted and unlimited multiplication of the ascomycete Podospora anserina. C R Hebd Seances Acad Sci 237(15): 838-840. PMID: 13107134

57. Osiewacz HD, Hamann A, Zintel S (2013). Assessing organismal aging in the filamentous fungus Podospora anserina. Methods Mol Biol 965(439-462. doi: 10.1007/978-1-62703239-1_29

58. Chaveroche MK, Ghigo JM, d'Enfert C (2000). A rapid method for efficient gene replacement in the filamentous fungus Aspergillus nidulans. Nucleic Acids Res 28(22): E97. PMID: 11071951

59. Lecellier G, Silar P (1994). Rapid methods for nucleic acids extraction from Petri dish-grown mycelia. Curr Genet 25(2): 122-123. PMID: 8087879

60. Luce K, Osiewacz HD (2009). Increasing organismal healthspan by enhancing mitochondrial protein quality control. Nat Cell Biol 11(7): 852-858. doi: 10.1038/ncb1893

61. Borghouts C, Kimpel E, Osiewacz HD (1997). Mitochondrial DNA rearrangements of Podospora anserina are under the control of the nuclear gene grisea. Proc Natl Acad Sci U S A 94(20): 10768-10773. doi: 10.1073/pnas.94.20.10768

62. Pfaffl MW (2001). A new mathematical model for relative quantification in real-time RT-PCR. Nucleic Acids Res 29(9): e45. PMID: 11328886

63. Wittig I, Braun HP, Schägger H (2006). Blue native PAGE. Nat Protoc 1(1): 418-428. doi: 10.1038/nprot.2006.62

64. Krause F, Scheckhuber CQ, Werner A, Rexroth S, Reifschneider NH, Dencher NA, Osiewacz HD (2004). Supramolecular organization of cytochrome $\mathrm{c}$ oxidase- and alternative oxidase-dependent respiratory chains in the filamentous fungus Podospora anserina. J Biol Chem 279(25): 26453-26461. doi: 10.1074/jbc.M402756200

65. Unger AK, Geimer S, Harner M, Neupert W, Westermann B (2017). Analysis of yeast mitochondria by electron microscopy. Methods Mol Biol 1567: 293-314. doi: 10.1007/978-1-49396824-4_18 